\title{
ES2019-3818
}

\section{DEVELOPMENT AND TEST OF A DIRECT CONTACT HEAT EXCHANGER (PARTICLE - AIR) FOR INDUSTRIAL PROCESS HEAT APPLICATIONS}

\author{
Johannes Hertel ${ }^{1}$, Miriam Ebert ${ }^{1}$, Lars Amsbeck ${ }^{1}$, Birgit Gobereit ${ }^{1}$, Jens Rheinländer ${ }^{1}$, \\ Alexander Hirt ${ }^{2}$, Cathy Frantz ${ }^{1}$ \\ ${ }^{1}$ Institute of Solar Research, German Aerospace Center, 70569 Stuttgart, Germany \\ Contact author: johannes.hertel@dlr.de \\ ${ }^{2}$ Biotechnology and Process Engineering, Furtwangen University, 78054 Villingen-Schwenningen
}

\begin{abstract}
A direct absorption receiver using ceramic particles (CentRec) has been successfully developed by DLR and tested under solar conditions at the Juelich Solar Power Tower, demonstrating receiver outlet temperatures of more than $900{ }^{\circ} \mathrm{C}$. The next step towards commercial application of the technology is to demonstrate a cost-effective, high temperature heat extraction and transfer to a process medium. Besides e.g. steam for electricity generation in a steam turbine, hot air can be used to supply heat to industrial processes with energy demand at high temperature level.

A great potential for higher efficiencies and lower costs has been identified for a moving bed heat exchanger. Several concepts of direct contact heat exchangers have been analyzed and evaluated. The selected concept is a combination of several crossflow-sections that are arranged in series with fluidmixing-chambers between each crossflow-section.

Based on the selected design a heat exchanger prototype with $10 \mathrm{~kW}$ thermal power and a design air outlet temperature of $750{ }^{\circ} \mathrm{C}$ has been built and integrated into a test setup.

The test setup provides particles at $900{ }^{\circ} \mathrm{C}$ that are heated up electrically inside a hopper on top of the heat exchanger. Hot particles are then moving downwards (moving bed) from the hopper through the direct contact heat exchanger driven by gravity. Cold air supplied by a compressor flows through the particle bed in cross-flow and is heated up. The hot air flow leaves the heat exchanger with a temperature of $750{ }^{\circ} \mathrm{C}$. The particle mass flow is controlled by an oscillating mass flow controller, positioned under the heat exchanger. The cold particles are collected in a container on the bottom. The particle cycle is closed by transporting them back to the hopper. A measurement and control system is implemented to carry out the tests.
\end{abstract}

The test setup has undergone successful commissioning in October and an extensive testing phase started in January 2019.

This paper presents the development and manufacturing as well as the successful commissioning of the heat exchanger prototype.

\section{NOMENCLATURE \\ DCHX direct contact heat exchanger \\ $\mathrm{V}_{\text {air,Ergun }} \quad$ fluid superficial velocity}

\section{INTRODUCTION}

A direct absorption receiver using ceramic particles (CentRec) has been successfully developed by DLR and tested under solar conditions at the Juelich Solar Power Tower, demonstrating receiver outlet temperatures of more than $900{ }^{\circ} \mathrm{C}$ [1]. The next step towards commercial application of the technology is to demonstrate a cost-effective, high temperature heat extraction and transfer to a process medium.

Therefore, DLR developed a direct contact heat exchanger (DCHX) to transfer the heat, stored in solid ceramic particles. An off-sun direct contact heat exchanger test setup is currently in commissioning at DLR in Stuttgart (Germany). The heat transfer from particles with $900{ }^{\circ} \mathrm{C}$ to air will be tested. The DCHX is designed to reach hot air temperatures up to $750^{\circ} \mathrm{C}$, with particle exit temperatures of approximately $200{ }^{\circ} \mathrm{C}$. The driving force for particle transport is gravity, and is controlled by a particle mass flow controller (see Fig. 2). Target particle mass flows for the test setup are 5 to $35 \mathrm{~g} / \mathrm{s}$. The air flow is limited to $175 \mathrm{~m}^{3} / \mathrm{h}$ by the capacity of the side channel compressor used in the test setup. 
The DCHX uses the same particles as the CentRec receiver with the following properties:

\begin{tabular}{|l|l|}
\hline Material & Sintered Bauxite \\
\hline Manufacture description & Saint-Gobain Proppants 16/30 \\
\hline Median particle diameter & $0.917 \mathrm{~mm} / 0.038$ inch \\
\hline Bulk density & $2.02 \mathrm{~g} / \mathrm{cc} / 125 \mathrm{lbs} / \mathrm{ft}^{3}$ \\
\hline
\end{tabular}

TABLE 1: PARTICLE PROPERTIES

\subsection{Identification of the Heat Exchanger Concept}

When identifying promising concepts for the heat transfer from particles to air at very high temperature level, it was decided to focus on direct heat exchanger concepts.

This is because the generation of hot air in indirect heat exchangers (tube or plate) requires high driving temperature and pressure differences. In addition, the low heat transfer coefficient of air and the high costs per heat transfer area result in high costs for indirect heat exchangers (due to high material and manufacturing costs of the high temperature materials).

On the other hand, direct contact heat exchangers with particles are characterized by the large surface area per volume provided by the particles, which could result in a significantly better technical and economic performance.

One conceptual weakness of the DCHX concept is that the different flows are not separated anymore by walls and therefore contamination can happen. Depending on the application this may be a problem, for example a certain dust load in the hot air might be critical. Where necessary, mitigation measures like ceramic filter candles or hot cyclones can be applied.

\section{Concept Selection}

In [2] DCHX concepts were categorized into seven types as seen in figure 1 .
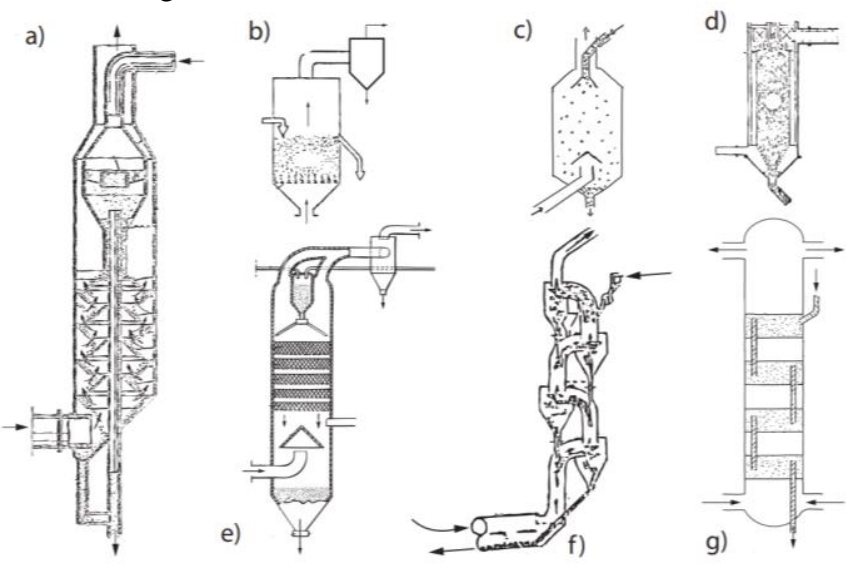

FIGURE 1: DCHX TYPES: A) CASCADING BED, B) SINGLE STAGED FLUIDIZED BED, C) FREE FALLING BED, D) MOVING BED, E) HINDERED FALLING BED, F) STAGED CYCLONES AND G), MULTI-STAGE FLUIDIZED BED [2]

Fluidized beds are used extensively in industry for example for refining (b) or as pre-heaters in cement and alumina kilns (f) but with significantly smaller particles as the ones used in CentRec (see table 1). All concepts using fluidized beds ( $b, f$ and $g$ ) were excluded during the concept selection due to the parasitic losses associated with the high fluidization velocities and resulting attrition needed for particle sizes above $1 \mathrm{~mm}$.

Falling particle concepts ( $\mathrm{a}, \mathrm{c}$ and e) are limited in the interaction time of particle/air heat exchange and have potentially attrition issues from the particle impacts, too. Therefore a moving bed type DCHX (d) was chosen.

One promising concept for moving bed type DCHX is being investigated by the Solar-Institut Jülich for many years, in which the air flows across a particle bed that is moving downwards [3]. Particle containment and open cross section for the air are provided by either a lamella wall or porous structures. More cross section for the air is provided compared to the counter-flow configuration in d).

That principal concept was chosen and adapted to a multistage cross flow DCHX.

\section{DCHX Concept}

The resulting design contains four segments of counterflow. The particle bed moves vertically in the DCHX in a framework of lamellas (see Fig. 2). The air flow streaming through the moving particle bed is heated subsequently within 4 passes. At every passage the heat is exchanged in a cross flow process. Mixing chambers are located after each pass in order to achieve a uniform temperature distribution. After the fourth pass the gas has reached the required air temperature.

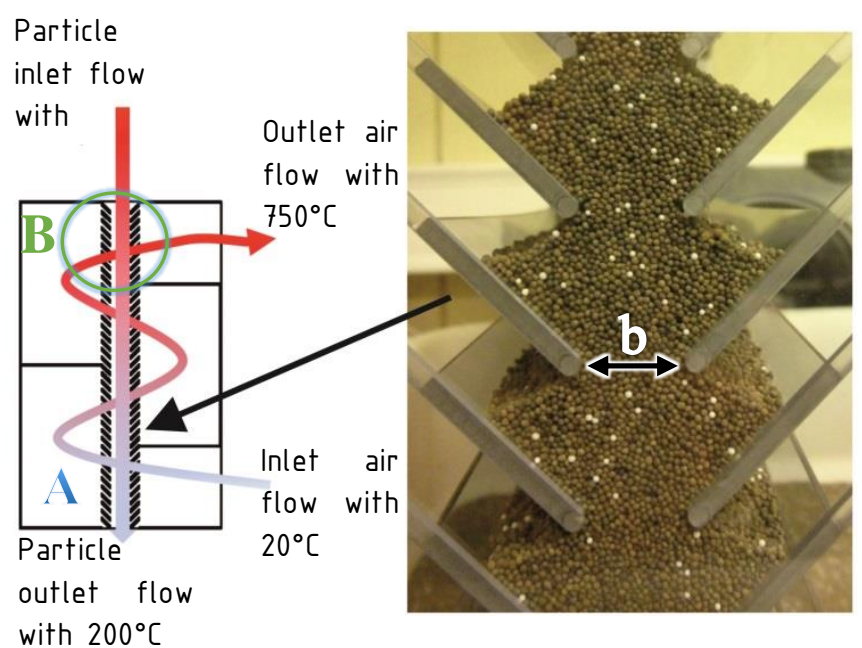

FIGURE 2: SCHEME OF THE WORKFLOW OF THE DCHX WITH MIXING-CHAMBERS (A) AND CROSSFLOW-SECTION (B) 


\subsection{Design and Manufacturing of the Heat Exchanger}

Based on the concept selection (described in 1.1) the DCHX was designed and detailed into technical drawings.

\section{Designing}

The challenge of moving bed type DCHX concepts is the "channeling effect", when small variations in local temperatures and porosity distributions lead to more air passing through a certain area, a "channel". Stronger cooling of the particles from the higher air mass flow in the channel results in lower particle temperatures in the channel and even higher air mass flows, a self-amplifying effect due to the strong temperature dependency of the air viscosity.

The dimensions of this multi-stage cross flow DCHX are governed by the maximum allowable air velocity at which particles would begin to be blown out of the lamellas. This velocity can be determined using the Ergun-Formula [4] to $\mathrm{v}_{\text {air,Ergun }}=0.289 \mathrm{~m} / \mathrm{s}$ for $900^{\circ} \mathrm{C}$, a sauter particle diameter of $0.000695 \mathrm{~mm}$, a porosity of 0.421 and a particle material density of $3490 \mathrm{~kg} / \mathrm{m}^{3}$ at ambient pressure.

The cross sections (for air flow in the channel) of the lamella walls were then accordingly selected in a way that the blow out conditions are not reached at the design value for the air mass flow.

The distance between the lamellas (see letter b in Fig. 2) is not governed by heat transfer aspects. Instead, the distance between the lamellas has to be large enough to safely avoid bridging of particles and resulting blockage of the moving bed channel.

Bridging can occur for gap widths up to 5 times the maximum particle diameter. With particles of up to $1.8 \mathrm{~mm}$ in the particle distribution and a high safety factor a gap size of $\mathrm{b}=16 \mathrm{~mm}$ between the lamellas was chosen.

The design results in the following properties of the heat exchanger:

\begin{tabular}{|l|l|}
\hline Air inlet temperature & $25^{\circ} \mathrm{C}$ \\
\hline Air outlet temperature & $750{ }^{\circ} \mathrm{C}$ \\
\hline Particle inlet temperature & $900{ }^{\circ} \mathrm{C}$ \\
\hline Particle outlet temperature & $200^{\circ} \mathrm{C}$ \\
\hline Air mass flow & $0.012 \mathrm{~kg} / \mathrm{s}$ \\
\hline Particle mass flow & $0.01 \mathrm{~kg} / \mathrm{s}$ \\
\hline Thermal Power of the DHCX & $10 \mathrm{~kW}$ \\
\hline Amount of lamellas & 40 \\
\hline Pressure drop (without filter) & $\sim 150 \mathrm{~Pa}$ \\
\hline
\end{tabular}

TABLE 2: PROPERTIES OF THE DHCX

The particle mass flow was determined by energy balance calculation considering the temperature values aimed for at air inlet and outlet and particle inlet and outlet as well as a fixed value for the air mass flow.

\section{Engineering}

The following chapter describes the detailed design of the heat exchanger. The heat exchanger consists of three main components:

- lamella wall, through which the air flows across a moving downwards particle bed

- baffles, which direct the flow of air through the heat exchanger

- housing, which serves as a support structure and prevents heat loss

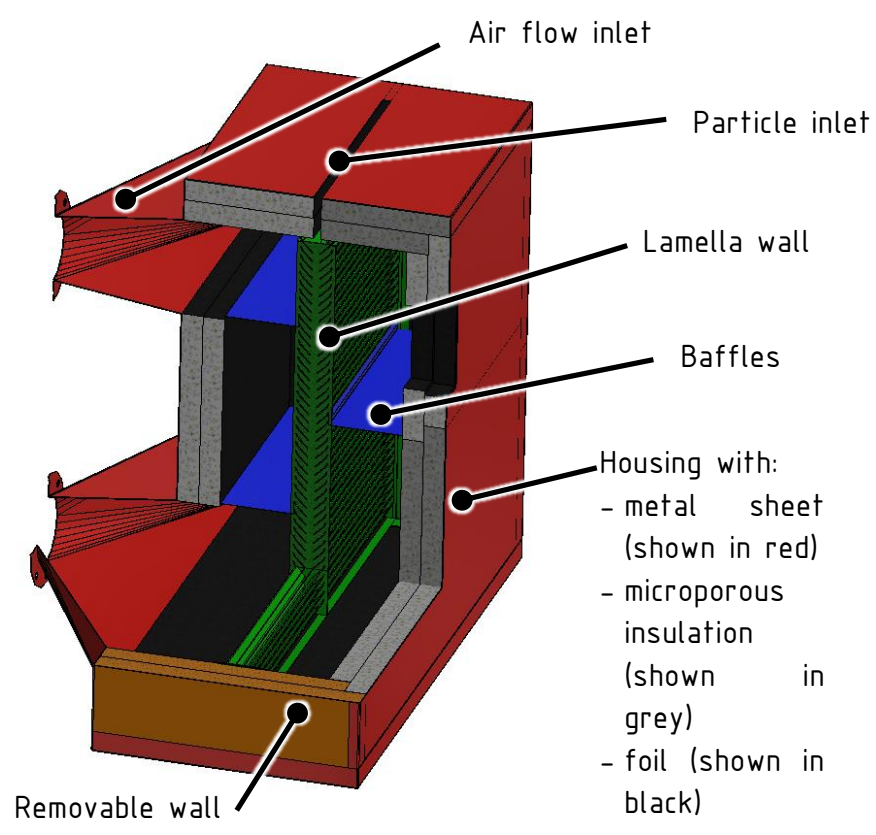

FIGURE 3: MAIN COMPONENTS OF THE HEAT EXCHANGER

\section{Lamella wall}

At design point conditions, the particles enter with $900{ }^{\circ} \mathrm{C}$ at the upper end of the lamellar wall and leave it at around $200{ }^{\circ} \mathrm{C}$ at the lower end. Since the large temperature difference can lead to high stresses on the lamellar wall, the design was verified by FEM yield analysis. The FEM model consists of the lamellas and three support structures that hold the lamellas and allow a flexible expansion of the lamellas as well as an easy replacement. The simulation takes into account the temperature loads as well as the weight of the particles. Due to symmetry characteristics only a half of the lamella wall was modelled. Fig. 4 shows the stress distribution on the lamella wall. The maximum stress of $27.5 \mathrm{MPa}$ only occurs at a few spots and can be neglected. 


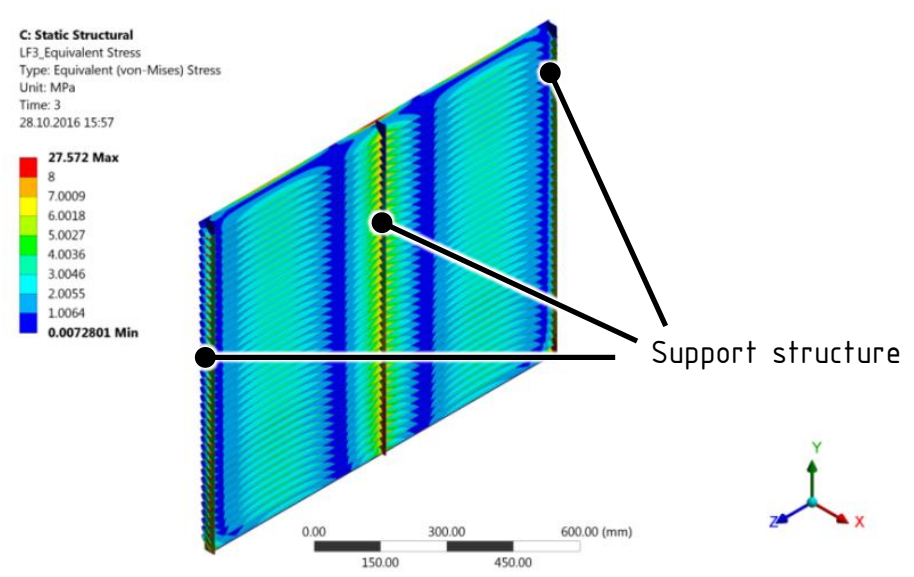

FIGURE 4: STRESSES ON THE WALL OF LAMELLAS UNDER THERMAL AND MECHANICAL LOAD (TEMPERATURE 200$900{ }^{\circ} \mathrm{C}$; WEIGHT FORCE OF PARTICLES)

As a result of the simulation, it was shown that the allowable creep rupture strength for the material 1.4828 (material designation) is not exceeded in 1000 hours of operating time. At 10,000 hours operating time this value is exceed for the topmost lamellas. The results show the design is well applicable for extensive prototype testing. When using the design for commercial applications, the topmost lamella can easily be replaced after 1000 hours or made of other materials with higher strength values, for example material 2.4856 (material designation) or composite sheet ceramic.

\section{Housing}

The housing consists of several layers listed in Table 3. The first layer is the outermost layer.

\begin{tabular}{|l|l|}
\hline 1st layer & $2 \mathrm{~mm}$ metal sheet (stainless steel) \\
\hline 2nd layer & $100 \mathrm{~mm}$ insulation (microporous insulation) \\
\hline 3rd layer & foil with ball embossing \\
\hline
\end{tabular}

TABLE 3: LAYERS OF THE HOUSING FROM THE DCHX

The first layer consists of a folded metal sheet which serves as a holding structure. The second layer consists of $100 \mathrm{~mm}$ thick microporous insulation. Microporous insulation has lower thermal conductivity compared to other insulation materials. As a result, the heat exchanger can be designed very compact. The third layer is a foil with a ball embossing that prevents the discharge of insulation material into the heat exchanger.

The housing design facilitates maintenance and inspection work through two removable side walls. Particle in- and outlet is realized by rectangular gaps on top and bottom of the housing. Connections for the air supply and discharge are integrated on one side wall of the housing.

\section{Baffles}

The baffles are used to guide the air flow and are made of foil with ball embossing. The embossing supports the stability of the foil. A foil of $0.1 \mathrm{~mm}$ was chosen, which can expand well at elevated temperatures without resulting in high stresses.

\subsection{Description of the Test Setup}

\section{Construction of the Test Setup}

In order to test the performance of the DCHX a test setup was build up.

Fig. 5 shows the core part of the test setup.
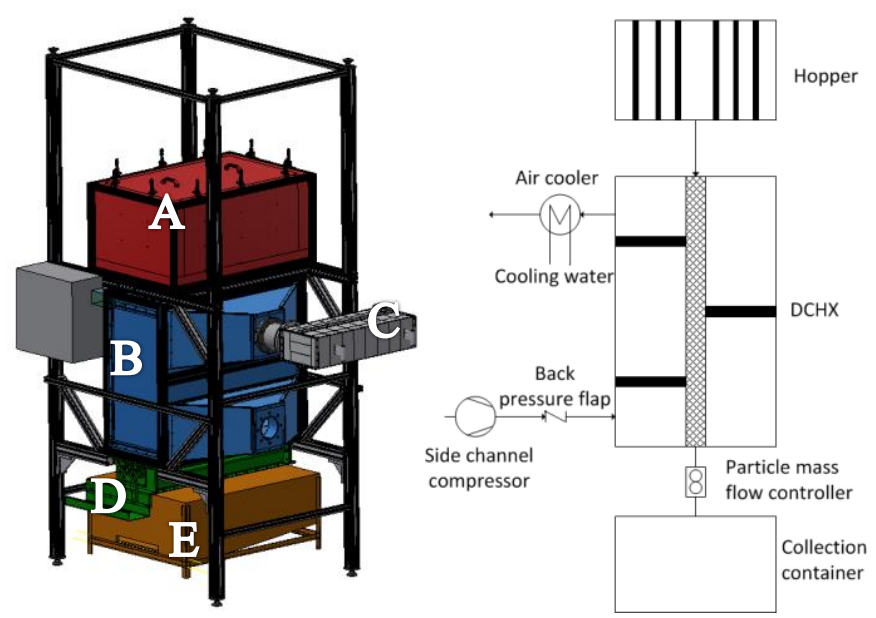

FIGURE 5: 3D MODEL AND FLOW SHEET OF THE TEST SETUP WITH HOPPER AND LID (A), DCHX (B), AIR COOLER (C), PARTICLE MASS FLOW CONTROLLER (D) AND COLLECTION CONTAINER (E).

\section{Function of the Test Setup}

Cold particles $\left(<200{ }^{\circ} \mathrm{C}\right)$ stored in the insulated collection container are supplied to the hopper. The particles are heated by electrical heating elements up to $900{ }^{\circ} \mathrm{C}$. Once the particle temperature has reached the value aimed for, the particle flow is started by activating the particle mass flow controller. The particles then flow through the DCHX, driven by gravity. For a new test run, the collection container, which is filled with the particles, is lifted with a pallet stacker to pour the particles back into the hopper. The DCHX will be tested in batch operation due to lack of space in the lab. In a commercial system a particle transport system that ensures a continuous particle mass flow will be used.

\section{Key Components of Test Setup}

\section{Hopper with Heater}

On top of the test setup is the hopper. With a volume of $0.33 \mathrm{~m}^{3}$ it has room for about $600 \mathrm{~kg}$ of particles. For the electric heating process 40 Ipermax cartridge heaters (Type HLP) with each a power of $1 \mathrm{~kW}$ are mounted vertically in the hopper. At the bottom of the hopper, an outlet slot leads into the DCHX. 


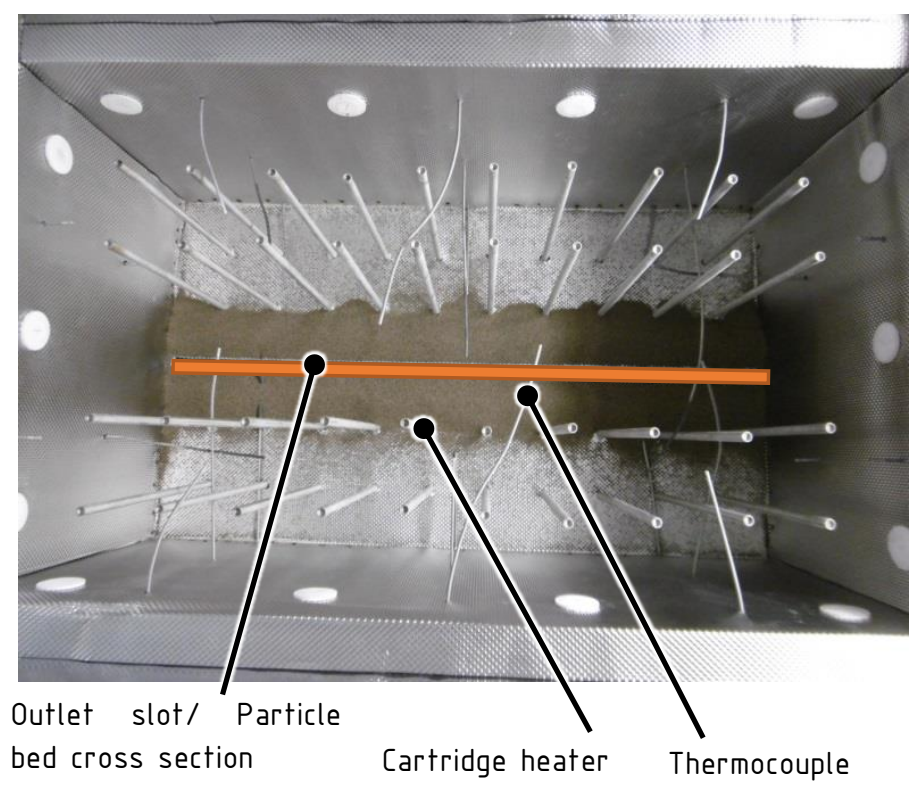

FIGURE 6: TOP VIEW PICTURE OF THE HOPPER WITH HEATING CARTRIDGE AND THERMOCOUPLES

\section{Air Cooler}

As the exhaust air is released to the environment, a water cooled tube coil heat exchanger is integrated at the DCHX air outlet. It cools the $750{ }^{\circ} \mathrm{C}$ hot air in a counter flow process down to less than $100^{\circ} \mathrm{C}$.

\section{Particle Mass Flow Controller - OSZILLOMAT®}

The particle mass flow is adjusted by a dosing device called "OSZILLOMAT®", which is mounted below the DCHX. The oscillating movement of two beams in the OSZILLOMAT creates a gap through which the particles emerge (see Fig. 7). The OSZILLOMAT was chosen because it ensures a homogeneous flow of the moving bed. A constant particle bed cross section (shown on Fig. 6) is ensured from the hopper down to the OSZILLOMAT. The thermal expansion of the lamella wall does not affect the particle flow. Only the gap width of the particle bed cross section at the OSZILLOMAT is important to ensure a constant particle flow. Therefore, the edges of the two OSZILLOMAT beams have to be very parallel to each other to ensure a constant flow of the particles in the DCHX.

The particles fall into the isolated collection container. A flexible compensator flanged to the OSZILLOMAT connects the dosing device to the collection container (not shown on the figure).

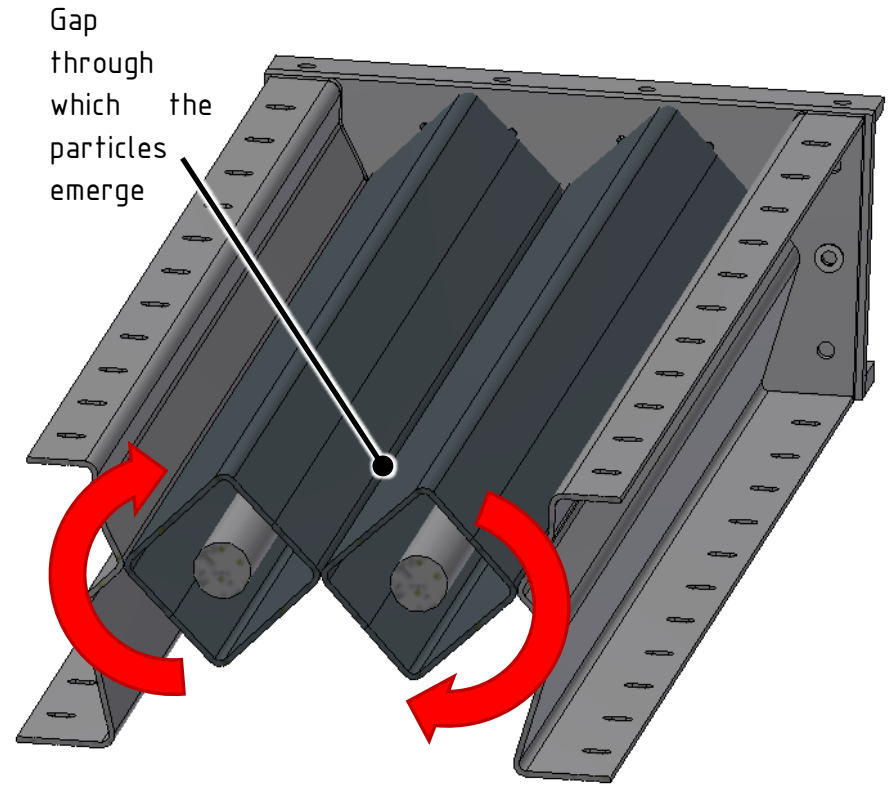

FIGURE 7: SCHEME OF THE OSZILLOMAT. THE ROTATION OF THE OSCILLATING BARS CREATES A GAP THROUGH WHICH PARTICLES EMERGE

\section{Transport}

Before each test run, the collection container filled with particles is lifted with a pallet stacker to pour the particles back into the hopper (see Fig. 8). Other transport options were excluded due to lack of space in the lab and to prevent particle attrition. As means of continuous transport with little to no attrition, bucket elevator or conveyor belt can be used in a commercial application. 


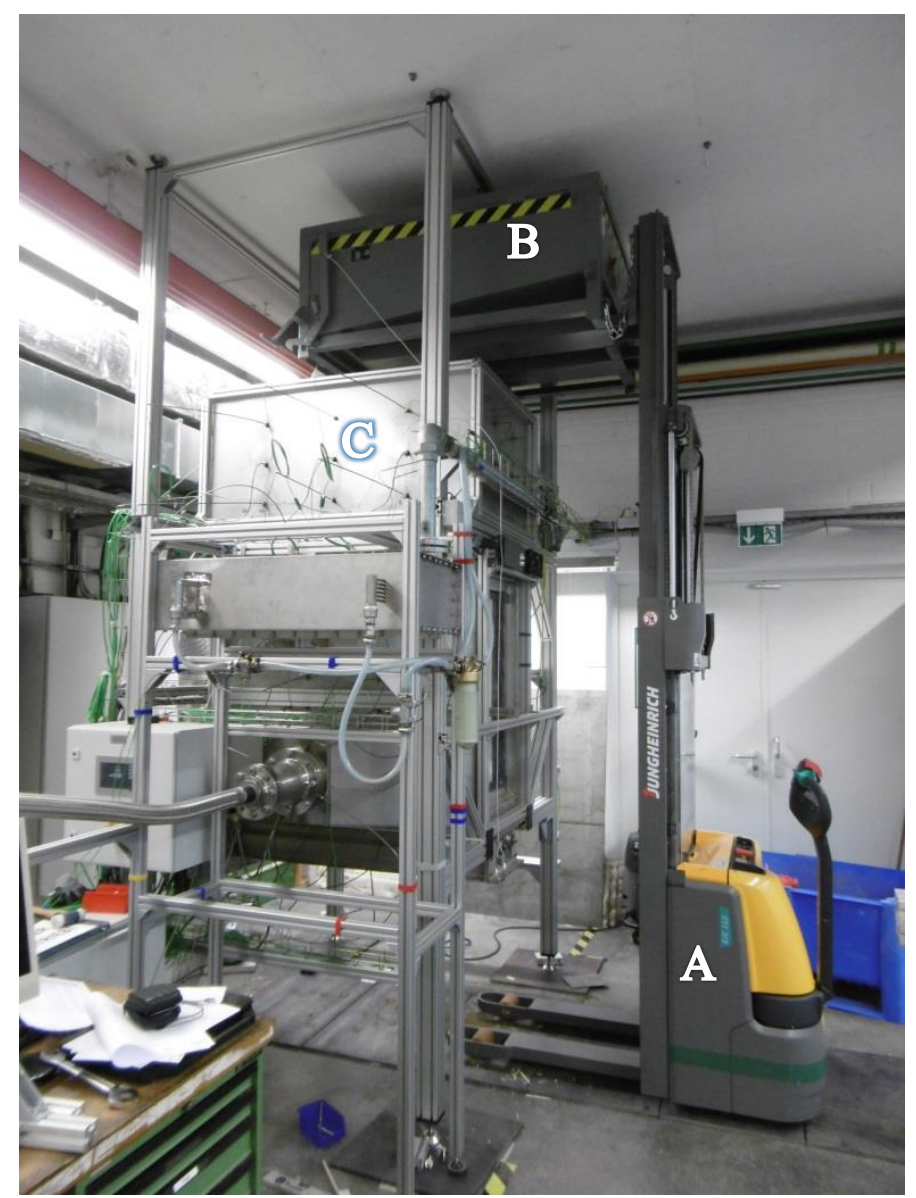

FIGURE 8: PICTURE SHOWING THE TRANSPORT SYSTEM USED FOR THE TEST SETUP. A PALLET STACKER (A) LIFTS THE PARTICLE-FILLED COLLECTION CONTAINER (B) TO THE HOPPER (C) TO SUPPLY THE PARTICLES INTO THE HOPPER.

\section{Measurement Instrumentation}

Several thermocouples are installed in the test setup. Each heating element in the hopper is controlled by one thermocouple. In addition there are 26 thermocouples mounted in the hopper in order to measure the bulk temperature profile in the tank. The air temperature in the 5 mixing chambers of the heat exchanger as well as the temperature of the moving particle bed is monitored with a total of 23 thermocouples. Furthermore both inlet and outlet temperature of the air and the particles from the heat exchanger are measured. To observe the heat loss in the hopper as well as the collection container, surface thermocouples are affixed to the lateral areas.

The incoming air mass flow into the heat exchanger is determined by an air mass flow meter.

To assess pressure losses in the heat exchanger, pressure sensors are mounted at the air inlet and outlet.

\subsection{Commissioning}

Cold commissioning started in October 2018 with an I/O signal check of all signals without particles. All measurement signals, control commands and the safety system were tested.

In the second step, the test setup was filled with particles. The test setup was filled from the hopper. During filling, it was observed that a few particles jump out from the lamellar to the baffle plates. Therefore, in later operation, the heat exchanger should never run dry and should always be filled with particles.

After particle filling, the air system was put into operation and the pressure drop of the heat exchanger was measured and compared to the calculated pressure loss. At a maximum air mass flow of approximately $175 \mathrm{~m}^{3} / \mathrm{h}$, the measured pressure loss is $11 \mathrm{mbar}$. The calculated pressure loss is $10 \mathrm{mbar}$. At the maximum air mass flow no blow out of particles from the lamellar wall could be observed.

During operation with air flow, leakages were detected at the DCHX, due to not hermetically sealed foil. The leaks were repaired with high temperature silicone or aluminum tape.

\section{OSZILLOMAT}

The particle mass flow is adjusted based on the size of the opening gap of the two oscillating beams and the waiting time.

The opening gap is automatically determined based on a setpoint input in percent. The waiting time is the closing time in which the beams of the oscillator are closed before reopening. The parameter can be set on the OSZILLOMAT control.

The leaving particle mass and the time were measured in order to define the two parameters and to reach the required particle mass flow of $10 \mathrm{~g} / \mathrm{s}$.

\subsection{Conclusion and Outlook}

The direct contact heat exchanger has been successfully designed, manufactured, assembled and put into operation. The heat exchanger was integrated into a test setup in order to test it under real conditions.

The test setup was built and put into operation. The cold commissioning is successfully completed. So far, no weak spots have been identified.

In the following experiments, the particle temperatures in the hopper will be increased gradually. The measured data are recorded and compared with the design data.

It is planned to use the developed heat exchanger in the EU-Project PreMa to preheat manganese ore with solar heat before it goes into a smelter.

\section{ACKNOWLEDGEMENTS}

The development of the CentRec ${ }^{\circledR}$ technology was supported by the Helmholtz Validation Fund (grant no. HVF0028) as well as by DLR Technology Marketing and DLR's Energy Programme Directorate (grant no. LRV 16/113). 


\section{REFERENCES}

[1] Ebert, Miriam. First On-Sun Tests of a Centrifugal Particle Receiver System, Power \& Energy ASME 2018.

[2] Green, H.J. ; Leboeuf, C.M. ; Bohn, M.S. ; Energy, United States. D. ; Institute, Solar Energy R.: Technical and Economic Evaluation of a Solid-particle/air Direct-contact Heat Exchanger. Solar Energy Research Institute (SERI) and
Sandia National Laboratories, 1986 (SERI/TR), prepared under Task No. 4254.1098 FTP No. 455

[3] Warerkar, S.; Schmitz, S.; Goettsche, J.; Hoffschmidt, B.; Reißel, M.; Tamme, R.: Air-Sand Heat Exchanger for

High-Temperature Storage, Journal of Solar Energy

Engineering, May 2011, Vol. 133

[4] VDI-Wärmeatlas, Laf, 10. Edition 2006 